\title{
ON THE EFFECT OF FINE-SCALE RADIAL VOID DISTRIBUTIONS ON ASSEMBLY CRITICALITY AND PIN POWER DISTRIBUTION
}

\author{
Dean Price ${ }^{1 *}$ Andrew Gacek ${ }^{2}$, Tomasz Kozlowski ${ }^{1}$, and Majdi I. Radaideh ${ }^{1}$ \\ ${ }^{1}$ Department of Nuclear, Plasma and Radiological Engineering, \\ University of Illinois at Urbana-Champaign, Urbana, IL 61801 \\ ${ }^{2}$ Department of Electrical and Computer Engineering, \\ University of Illinois at Urbana-Champaign, Urbana, IL 61801
}

deanrp2@illinois.edu, andrewg3@illinois.edu, txk@illinois.edu, radaideh2@illinois.edu

\begin{abstract}
The assumption that void fraction, and by extension coolant density, is uniform in the radial direction is a common approximation used in lattice physics simulations. In this study, models without uniform radial void fraction are used and lattice criticality and pin powers are investigated in two ways. One way uses hypothetical models that reflect extreme radial void distributions; modifications such as uniform radial pin enrichment and the removal of gadolinium rods are included in these models as well. Experimentallydetermined boiling water reactor radial void distributions are also replicated in neutronics models using Serpent 2. It is found in the hypothetical models that the presence of gadolinium rods has a large effect on the interaction between lattice criticality and radial void distribution. It was also found that considering experimental radial void fraction distributions had the largest effect on the pin power of the rods containing gadolinium. Furthermore, it is observed that considering realistic radial void distributions, in general, decreased lattice criticality. The reason can be attributed that to the passive negativefeedback design of light water reactors. These are useful findings because calculation of more accurate peaking factors can lead to efficient and yet safer reactor operation.
\end{abstract}

KEYWORDS: Boiling Water Reactor, Criticality, Radial Void Distribution, Serpent 2

\section{INTRODUCTION}

Whenever a real-world system is modeled, certain assumptions are inevitable to simplify the model structure and make it manageable. For example, in criticality safety applications, the conservative approach considers fuel to be fresh to avoid expensive depletion calculations. However, it is important to investigate these assumptions to quantify their effect on results. In reference to spent fuel criticality, the results given in [1] demonstrate that that this assumption is not always valid and that

\footnotetext{
${ }^{*}$ Corresponding author
} 
in the case of boiling water reactors (BWRs), this assumption actually leads to a large underestimation in spent fuel criticality. In this paper, the effect of radially-averaging void distribution to a constant coolant density is investigated. Past work has explored the effect of axial coolant density distribution in BWRs [2,3], so it will not be addressed here.

One comprehensive study [4] captured a realistic radial void distribution and gauge the effect on reactor parameters. It intended to capture the uncertainty associated with conventional homogeneous approximations by thoroughly considering variations in void fraction distributions at the subchannel level. The subchannel thermal hydraulics code COBRAG and neutronics code TGBLA (or MCNP4C) were coupled in an iterative method to capture the interaction between pin power and coolant density. Some important results relevant to the present study were found. First, it was found that the heterogeneous void distribution flattened the fission density distribution. The authors asserted that this was because areas of higher fission density would result in a greater void fraction due to the higher heat generation in those areas. Quantitatively, it was found that the rod power peaking factor could be reduced by up to $4 \%$. It was also found that $\mathrm{k}_{\infty}$ decreased due to the realistic void distribution, this was attributed to an increased gadolinium rod worth.

In this study, no coupling will be performed. Instead, experimentally-determined radial void distributions will be compared to an equivalent case with a radially averaged void distribution. Also, extreme cases will be used to investigate the extent that lattice criticality can change for a given void fraction. These cases are not intended to be realistic, they are supposed to be a launching point for further investigation. Lattice criticality and pin power distributions will be investigated using Serpent 2 [5] with the ENDF/B-VII.0 libraries [6].

\section{METHODOLOGY}

This section is divided into 3 subsections, each intended be concise descriptions of 1) the models used in this study 2) hypothetical cases used to determine the effect of radial void distributions while the models are still simple and 3) integration of experimentally-determined radial void distributions into neutronics models.

\subsection{Models}

All analysis in this study will be based upon the 2F2DN23 assembly, originally irradiated in Fukushima-Daini-2 unit [7]. This model was selected because it has been used in many validation studies $[8,9,3]$, which means there is thorough documentation on its geometry and fuel composition. Also, it follows the same geometry as one of the assemblies used in the BWR Fullsize Finemesh Bundle Test (BFBT) benchmark, see Table 2.3.1 in [10]. This benchmark will be used to test the effect of experimentally-determined radial void distributions on assembly criticality. The 2F2DN23 is a Hitachi $8 \times 8$ BWR assembly with 8 rods containing $4.5 \mathrm{wt} \% \mathrm{Gd}_{2} \mathrm{O}_{3}$ and an average radial enrichment of $3.23 \%$. In this study, the assembly is modeled in $2 \mathrm{D}$ and assumed to have the fuel enrichment and composition given in Figure 1a. The fuel composition used in the assembly is assumed to be fresh. The statistical uncertainty associated with calculations is kept below $20 \mathrm{pcm}$ uncertainty in $k$. 


\subsection{Simulation of Radial Void Distributions}

In order to understand the effect of radial void distribution on assembly criticality, some hypothetical cases will be investigated. These hypothetical cases will help in determining the effect of radial void distributions, while maintaining simple models. Figure $1 \mathrm{~b}$ gives a graphical depiction of the three cases considered. These cases have their coolant regions divided into two: one region containing completely water vapor and the other completely containing liquid water. The first case is the "hollow" case, this case has a rounded-corner square boundary centered on the assembly that divides the liquid and vapor regions. This boundary is filled with void. The second case is the "full case". This case is similar to the hollow case except instead of the void being at the center of the assembly, it fills the periphery. In each case, the boundary can change positions in order to adjust the fraction of the assembly filled with void. These cases are not intended to represent any realistic scenario in an operating reactor, they are only included to provide bounding estimates and to see how assembly $k$ changes in cases of extreme heterogeneity.

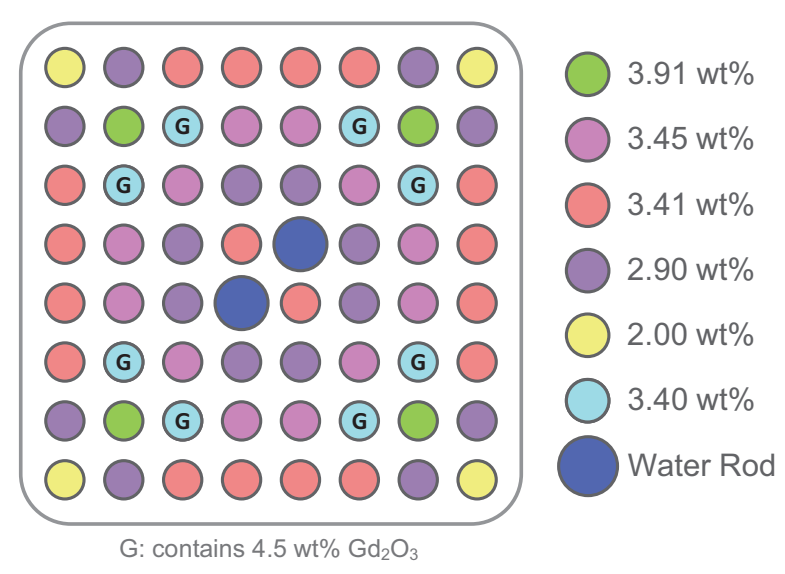

(a)

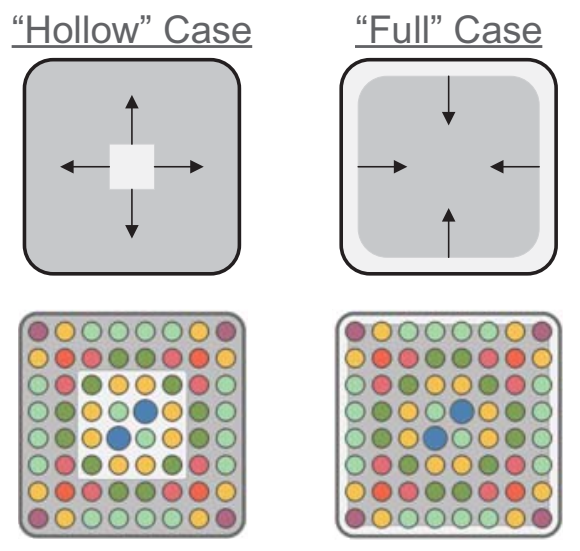

(b)

Figure 1: (a) Radial enrichment layout of Fukushima Daini 2: assembly 2F2DN23. (b) Graphical depiction of hypothetical cases with sample geometries to show how void is represented in each case. Dark shades indicate areas of liquid while lighter shades indicate areas of void.

These cases will be compared to a case that has a uniform radial coolant density. Throughout this study, a case with uniform radial coolant density acts as a basis for comparison because it is the modeling method that most studies use to represent void fraction. During each calculation, only the density of the coolant within the assembly channel is adjusted according to Equation 1. For these hypothetical cases, $\rho_{c}$ is the resulting coolant density when considering a liquid density $\left(\rho_{l}\right)$ of $0.7401 \mathrm{~g} / \mathrm{cm}^{3}$ and a vapor density $\left(\rho_{v}\right)$ of $0.0365 \mathrm{~g} / \mathrm{cm}^{3}$ at some void fraction $\alpha$. Also, further modifications to these hypothetical cases will be made to explore the effect of averaging radial pin enrichment and removing gadolinia from the lattice model.

$$
\rho_{c}=\rho_{l}(1-\alpha)+\rho_{v}(\alpha)
$$


The next portion of analysis involves integrating experimentally-determined radial void distributions into neutronics models. Inoue et al. [11] performed a comprehensive experimental study that yielded radial void distributions with a resolution on the $0.03 \mathrm{~cm} \times 0.03 \mathrm{~cm}$ scale. These data were released as part of the BFBT benchmark [10]. In short, the benchmark included a channel-scale analysis of the radial void distributions using CT scanners.

Energy was generated in the pins using electric heaters. Two categories of radial pin powers were considered: (1) uniform pin power distribution, and (2) simulation pattern for beginning of operation. The uniform pin power distribution is simply each heater producing the same amount of heat. The simulation pattern was generated to emulate a realistic pin power distribution in a reactor.

For this analysis, these radial void distributions will be processed and then replicated in Serpent 2 to determine the effect of these void distributions on pin power and lattice criticality. Figure 2 is included to illustrate how the experimental data is replicated in a Serpent 2 model and Section 2.3 gives more details on this process. For analysis, these models are compared to a homogeneous model with a coolant density corresponding to the average coolant density in the coolant area of the heterogeneous model.

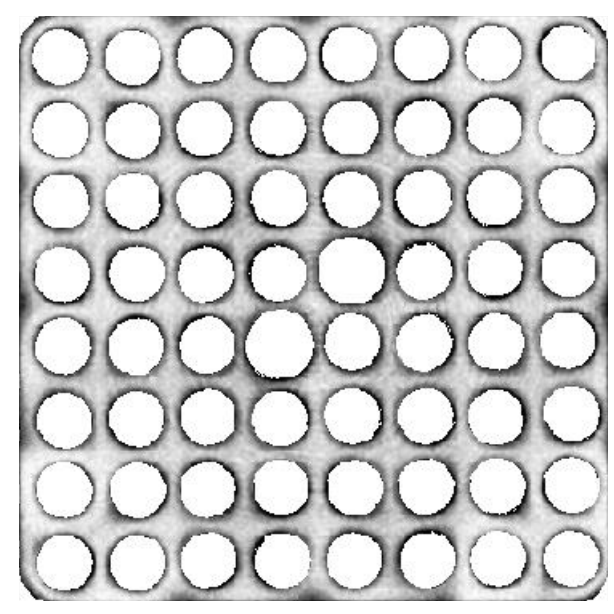

(a)

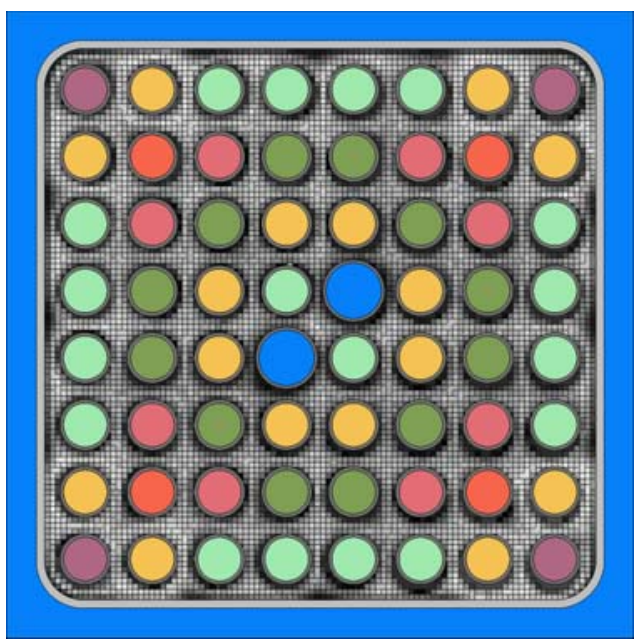

(b)

Figure 2: (a) Example of experimental data layout, lighter areas indicate areas of higher void fraction. (b) Example of processed Serpent 2 model based on same experiment.

\subsection{Experimental Data Processing}

Integrating the experimental data into Serpent models presents a set of unique challenges. First, the $2 \mathrm{D}$ void map data from the CT scans needed to be rotated and cropped. Coarsening to a 100 $\times 100$ grid using bilinear interpolation was done after this to ensure that cropping had no effect on final array size. From here, each cell is converted from a void fraction to a coolant density based on the pressure given for the experiment considered. The original experimental data did not include values for void in areas that the fuel pin is expected to occupy. Therefore, these areas are filled 
in with the average value around their perimeter because there is not perfect overlap between pin placement of the model and experimental data.

Following this, an equispaced $100 \times 100$ grid was created in the coolant region in a Serpent 2 model and each cell in the grid was filled with the corresponding coolant density from the experimental data. One particular challenge that arises from this is that due to pin overlap in the Serpent model, simply averaging the experimental void array does not yield the average void in the coolant region of the Serpent model. As such, a Monte Carlo sampling method which sampled random points in the coolant region and determined the corresponding density was used to generate radially averaged densities.

\section{RESULTS}

First, results and conclusions regarding the hypothetical cases will be presented. Conclusions regarding the sensitivity of $k$ to certain coolant regions are drawn and further work is described. Next, a discussion of the results around the experimental distributions are presented.

\subsection{Hypothetical Cases}

The criticality of the hypothetical cases depicted in Figure $1 \mathrm{~b}$ as a function of void fraction are included in Figure 3. These results also include cases where the gadolinium is removed from the lattice and the radial pin enrichment is averaged. For the normal lattice, the maximum deviation of the hollow case from the homogeneous case reaches about $2500 \mathrm{pcm}$. The same can be said for the full case, whose maximum deviation from the homogeneous case reaches about $1000 \mathrm{pcm}$. By examining the model geometry, this maximum deviation occurs as the boundary between the liquid and void region passes over the gadolinium rods; indicating the moderation of these rods tends to have a significant effect on lattice criticality. Also, for the hollow case, lattice criticality increases as void increases from around $25 \%$ to $45 \%$ void. This can all be attributed to moderation of the gadolinium rod. When the void fraction around the gadolinium rod is low, the worth of the gadolinium is low as well. As the boundary passes over the gadolinium rod, this rod worth is significantly changed, leading to the more expected negative void coefficient behavior shown from $45 \%$ on.

This assertion is further strengthened by the observation that these extreme deviations disappear when removing the gadolinium from the model. Furthermore, the lattice with uniform enrichment and gadolinium removed was included to gauge the effect of the interaction between pin enrichment heterogeneity and the void boundary. It is clear from this figure that after removing gadolinium, deviations from the homogeneous case are minimally effected by further averaging heterogeneous radial enrichment. From this analysis, further work on a subchannel-level sensitivity analysis would be in order. These results indicate that lattice criticality is most sensitive to coolant regions surrounding gadolinum rods.

\subsection{Simulation of Experimental Distributions}

Next, this line of inquiry is intended to be focused on capturing the effect of experimentally determined radial void distributions on lattice parameters. The data here uses experimental distributions 

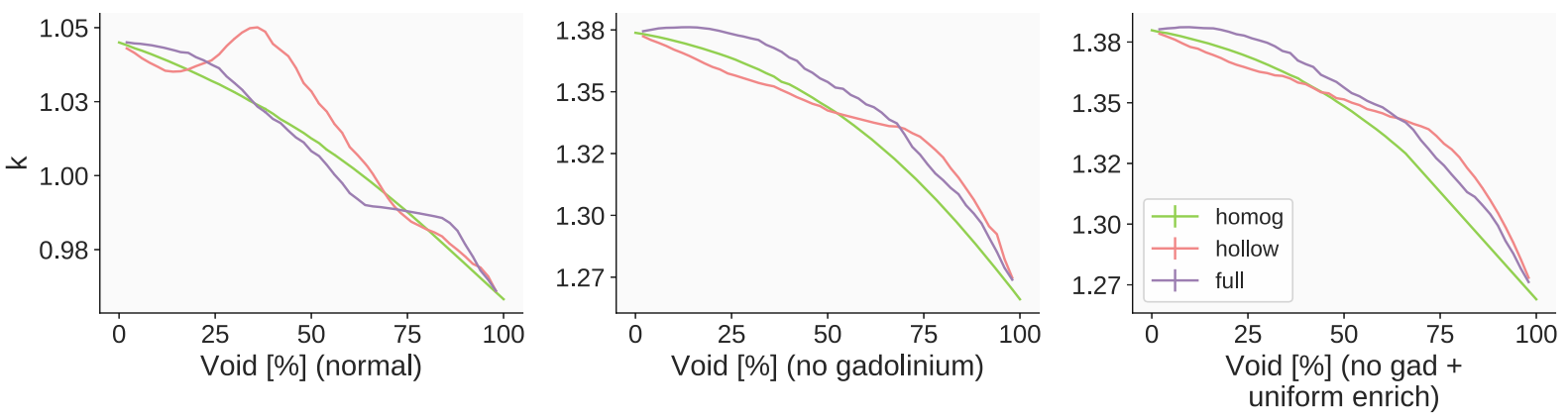

Figure 3: Criticality as a function of void for a normal lattice, a lattice with gadolinium removed and a lattice with uniform enrichment.

that come to be between 50 and $60 \%$ void when they are averaged radially. Both lattice criticality at beginning of cycle and pin power distributions, as shown in Figure 4 are investigated. From the left plot, the heterogeneous models consistently yield a lower $k$ than the homogeneous models. The dependence of this $\Delta k$ on void fraction is difficult to determine from this selected range of cases. However, it can be seen that the radial void distributions coming from the experiment with uniform pin powers more consistently has a larger criticality associated with the homogeneous case than the heterogeneous one. Also, these results indicate that conventional calculations based on homogeneous models yield conservative results for lattice operation. On the right side of Figure 5, the mean squared difference in pin powers from the homogeneous and heterogeneous case is shown. The largest discrepancy occurs in the pins containing $\mathrm{Gd}_{2} \mathrm{O}_{3}$. This is likely because the lower power pin in these rods during experiment would have a significant effect on the void fraction local to these rods. This effect is not captured in the homogeneous case. Figure 5 gives a comparison of the criticality of the homogeneous and heterogeneous models, distributions experimentally generated using uniform and simulated pin powers are separated. This figure also shows the degree of variability between the relationship in $k_{\text {homog }}$ and $k_{\text {hetero }}$. In order to further explore this assertion, more samples should be run in the future over a wider variety of void fractions.

Nevertheless, it is thought that these underestimations are the results of the negative-feedback aspect of BWRs. As a pin becomes better-moderated, it increases its power, thereby increasing the local void fraction. This then decreases the moderation of the pin, decreasing its power. Future work will run simulations at higher fidelity to explore this effect.

\section{CONCLUSIONS}

This study included hypothetical cases intended to explore the interaction of various lattice parameters such as gadolinium rods and heterogeneous radial pin enrichment with extreme void distributions. These cases were not intended to be realistic but instead used as a launching point for more advanced models. It was found that deviations from a case with uniform coolant density were maximized when the boundary between a region containing all liquid and a region containing all vapor passed over the gadolinium rods. This was true for both hypothetical cases considered. These results call for further analysis on the sensitivity of various lattice parameters to the void 

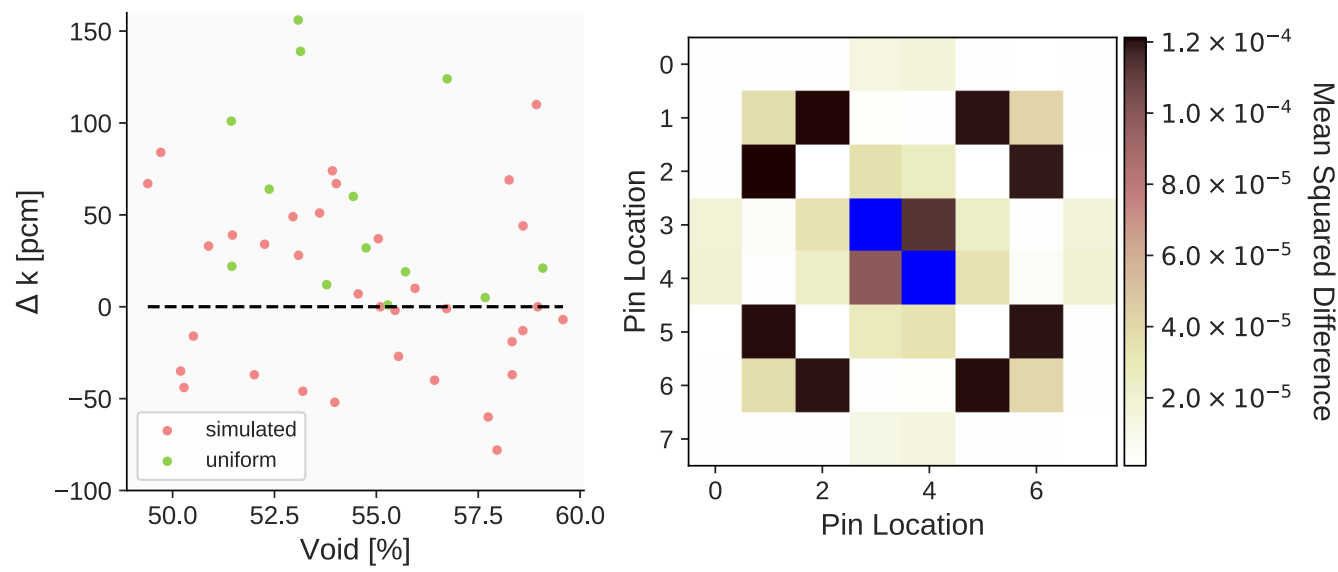

Figure 4: Comparison of experimentally-determined radial void distributions and their homogeneous equivalents. $k_{\text {homog }}-k_{\text {hetero }}$ (left) and mean squared difference of normalized pin powers between homogeneous and heterogeneous cases (right).
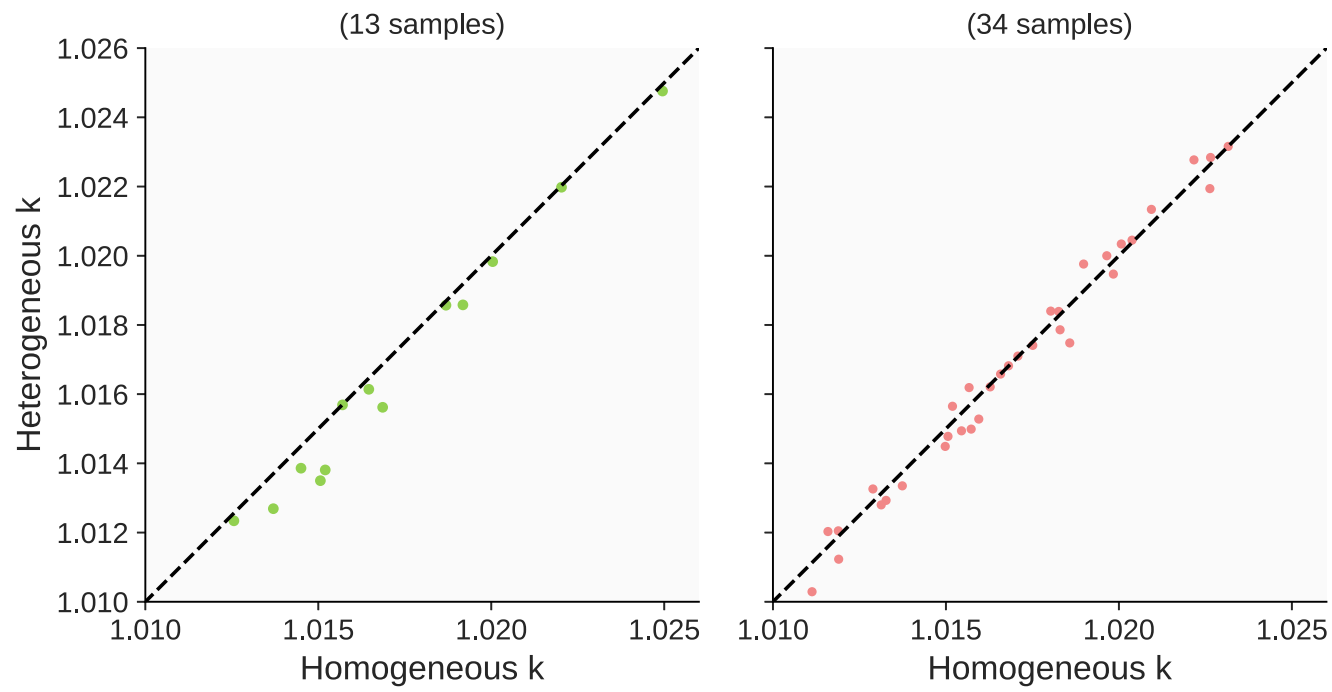

Figure 5: Comparison of experimentally-determined radial void distributions and their homogeneous equivalents. Distributions reflecting uniform pin powers (left) and distributions reflecting simulated pin powers (right).

fraction of different regions of the coolant. Also, for some configurations, criticality increased with increasing void, it is expected that this is due to an increasing gadolinium rod worth in these scenarios. This would be useful, because when coupled with the uncertainty in void fraction from experiments, some uncertainty in $k$ could be determined.

This study also seeks to incorporate experimentally-determined radial void fraction distributions into neutronics analyses. Both pin peaking factors and lattice criticality are investigated using 2D 
models and data from the BFBT benchmark. It is found that considering realistic radial void distributions usually decreases the criticality of the lattice model. These results agree with the results given in previous studies. The negative-feedback nature of light water reactors is an explanation for this behavior. Further work here should go into using higher fidelity models over a wider range of void distributions/flow regimes.

\section{REFERENCES}

[1] M. I. Radaideh, D. Price, and T. Kozlowski. "Criticality and uncertainty assessment of assembly misloading in BWR transportation cask." Annals of Nuclear Energy, volume 113, pp. 1 - 14 (2018).

[2] M. I. Radaideh, D. Price, D. O'Grady, and T. Kozlowski. "Advanced BWR criticality safety part I: Model development, model benchmarking, and depletion with uncertainty analysis." Progress in Nuclear Energy, volume 113, pp. 230 - 246 (2019).

[3] D. Price, M. I. Radaideh, D. O'Grady, and T. Kozlowski. "Advanced BWR criticality safety part II: Cask criticality, burnup credit, sensitivity, and uncertainty analyses." Progress in Nuclear Energy, volume 115, pp. 126-139 (2019).

[4] T. Ikehara, Y. Kudo, M. Tamitani, and M. Yamamoto. "Effect of subchannel void fraction distribution on lattice physics parameters for boiling water reactor fuel bundles." Journal of Nuclear Science and Technology, volume 45(12), pp. 1237-1251 (2008).

[5] J. Leppänen, M. Pusa, T. Viitanen, V. Valtavirta, and T. Kaltiaisenaho. "The Serpent Monte Carlo code: Status, development and applications in 2013." Annals of Nuclear Energy, volume 82, pp. 142-150 (2015).

[6] M. Chadwick, P. Obložinskỳ, M. Herman, N. Greene, R. McKnight, D. Smith, P. Young, R. MacFarlane, G. Hale, S. Frankle, et al. "ENDF/B-VII. 0: next generation evaluated nuclear data library for nuclear science and technology." Nuclear data sheets, volume 107(12), pp. 2931-3060 (2006).

[7] Y. Nakahara, K. Suyama, J. Inagawa, R. Nagaishi, S. Kurosawa, N. Kohno, M. Onuki, and H. Mochizuki. "Nuclide composition benchmark data set for verifying burnup codes on spent light water reactor fuels." Nuclear Technology, volume 137(2), pp. 111-126 (2002).

[8] U. Mertyurek, M. W. Francis, and I. C. Gauld. "SCALE 5 analysis of BWR spent nuclear fuel isotopic compositions for safety studies." Technical report, ORNL/TM-2010/286, Oak Ridge National Laboratory (2010).

[9] K. Tada, T. Kikuchi, T. Sakino, and K. Suyama. "Analyses of used BWR fuel assay data with the integrated burnup code system SWAT4. 0." Journal of Nuclear Science and Technology, volume 55(2), pp. 138-150 (2018).

[10] B. Neykov, F. Aydogan, L. Hochreiter, K. Ivanov, H. Utsuno, F. Kasahara, E. Sartori, and M. Martin. "NUPEC BWR full-size fine-mesh bundle test (BFBT) benchmark." Technical report, NEA/NSC/DOC(2005)5, OECD Nuclear Energy Agency, France (2006).

[11] A. Inoue, T. Kurosu, T. Aoki, M. Yagi, T. Mitsutake, and S.-i. Morooka. "Void fraction distribution in BWR fuel assembly and evaluation of subchannel code." Journal of Nuclear science and Technology, volume 32(7), pp. 629-640 (1995). 